Original Article

\title{
Effect of the Masako maneuver and neuromuscular electrical stimulation on the improvement of swallowing function in patients with dysphagia caused by stroke
}

\author{
HaEwon Byeon, DrSc ${ }^{1)}$ \\ 1) Department of Speech Language Pathology, Nambu University: 23 Cheomdan Jungangro, \\ Gwangsan-gu, Gwangju 506-706, Republic of Korea
}

\begin{abstract}
Purpose] The aim of this study was to compare improvements in swallowing function by the intervention of the Masako maneuver and neuromuscular electrical stimulation in patients with dysphagia caused by stroke. [Subjects and Methods] The Masako maneuver $(\mathrm{n}=23)$ and neuromuscular electrical stimulation $(\mathrm{n}=24)$ were conducted in 47 patients with dysphagia caused by stroke over a period of 4 weeks. Swallowing recovery was recorded using the functional dysphagia scale based on videofluoroscopic studies. [Results] Mean functional dysphagia scale values for the Masako maneuver and neuromuscular electrical stimulation groups decreased after the treatments. However, the pre-post functional dysphagia scale values showed no statistically significant differences between the groups. [Conclusion] The Masako maneuver and neuromuscular electrical stimulation each showed significant effects on the improvement of swallowing function for the patients with dysphagia caused by stroke, but no significant difference was observed between the two treatment methods.

Key words: Masako maneuver, Neuromuscular electrical stimulation, Functional dysphagia scale
\end{abstract}

(This article was submitted Feb. 9, 2016, and was accepted Apr. 7, 2016)

\section{INTRODUCTION}

Stroke is a disease in which provision of oxygen or nutrition to central or peripheral nerves is discontinued because of cerebrovascular infarction or hemorrhage. This disease is one of the major causes of death in Korea, along with cancer and heart disease. According to Statistics Korea, the number of deaths from cerebrovascular disease was 24,486 in 2014, a 4.2\% decrease from that in 2013; the prevalence rate of stroke is increasing as Korea is becoming an aged society ${ }^{1)}$.

Despite successful surgical treatment, stroke patients experience various aftereffects, such as motor and sensory disorders $^{2}$, and approximately $60 \%$ of stroke survivors suffer from dysphagia ${ }^{3)}$. Lack or loss of functions of swallowing muscles cause difficulty in swallowing of food boluses, which leads to deficiency in provision of nutrition. Moreover, continued dysphagia progresses into complications, such as aspiration and pneumonia, which lead to eventual death ${ }^{4}$. Thus, early detection and effective treatment of swallowing disorder are important to prevent complications.

The Masako maneuver and neuromuscular electrical stimulation (NMES) are swallowing rehabilitation techniques to improve problems of swallowing functions caused by stroke. The Masako maneuver is an oro-pharyngeal exercise rehabilitation technique to enhance the function of the constrictor phargeus superior. This technique is mainly performed to strengthen its function of pushing food boluses from the oral cavity to the pharynx by strengthening the contact between the tongue base and the laryngo-pharyngeal wall ${ }^{5}$. Recently, NMES has been developed and is a widely used rehabilitation technique that improves swallowing muscles in the pharyngeal stage ${ }^{6)}$. Numerous studies have reported treatment effects of NMES, 
but the results remain inconsistent ${ }^{7-9)}$. This study was conducted to compare improvements in swallowing function by the intervention of the Masako maneuver and NMES in patients with dysphagia caused by stroke.

\section{SUBJECTS AND METHODS}

A total of 47 patients who were diagnosed with dysphagia caused by stroke were included in the study. These patients underwent rehabilitation at two general hospitals in Inchon from June 2014 to December 2014 (Table 1). In accordance with the ethical standards of the Declaration of Helsinki, study objectives were explained to all patients and written informed consent was obtained prior to their participation. This study had adequate number of samples based on power analysis; the minimum number of samples was 45 with significance level $(\alpha)$ of 0.05 , effect size of 0.5 , and power of test (1- $\beta$ ) of 0.95 on the standard t-distribution. Selection standards for the subjects were dysphagia diagnosis of less than 6 months, no severe communication disorders, no depression, no nasogastric tube (L-tube), and no cognitive disorder (a score on the Korean version of mini-mental state examination of 24 or below).

The NMES group received a 20-minute stimulation per day, 5 days per week for 4 weeks by a swallowing therapist with VitalStim certification using VitalStim ${ }^{\circledR}$ (DJO Company, AP2116, USA), with a total of 15 treatments. Referring to Freed et al. ${ }^{7)}$, electrodes were attached on the mylohyoid and thyrohyoid muscles, and vibration frequency was set at $80 \mathrm{~Hz}$ with a width of $300 \mathrm{~ms}$.

The Masako maneuver, an exercise for swallowing rehabilitation to improve the function of the pharynx rear wall, was conducted for 20 minutes per day, 5 days a week for 4 weeks. This maneuver was performed by inducing dry swallowing. In this method, the patients softly bite the end of their tongues with their front teeth and maintain this posture while swallowing.

Swallowing recovery was recorded with the functional dysphagia scale (FDS) based on videofluoroscopic studies ${ }^{10)}$. The FDS, which is a 100-point evaluation scale, is used to identify overall swallowing problems, such as aspiration observed. A higher FDS score indicates worse swallowing function.

For analysis, a preliminary homogeneity test was conducted with independent sample t-test or $\chi^{2}$ test. Pre-post efficiency test was also performed with paired-sample t-test.

\section{RESULTS}

In the homogeneity test, no significant differences were observed in gender, time since dysphagia, or mean values of FDS (Table 1). Analysis of pre-post values of FDS of the Masako maneuver and NMES groups by using paired t-test (Table 2) showed no significant difference between the two groups. However, the mean values of FDS after treatment in both groups decreased.

\section{DISCUSSION}

Both Masako maneuver and NMES significantly improved the swallowing function in patients with dysphagia caused by stroke; this result agrees with those obtained by previous studies ${ }^{5-8)}$. The Masako maneuver, which is an exercise for swallowing rehabilitation to improve the function of pharynx constriction by strengthening muscle strength of the tongue base, has been reported to improve swallowing by helping the coordination of the larynx and the hyoid bone and improving the constriction of the pharynx and airway obstruction during pharyngeal swallowing ${ }^{6}$. NMES is a method of enhancing strength of the muscles with weak constriction by using functional electrical stimulation. This method is known to protect the airway by moving the hyoid bone through constriction of the suprahyoid muscle and raising the larynx together with the thyrohyoid muscle ${ }^{7-9}$. Several studies have explained the mechanism of NMES by the effect of the neural path related to swallowing and neuroplasticity of the brain ${ }^{11)}$. In particular, Freed et al. ${ }^{7)}$ emphasized that NMES is more effective in improving oral and pharyngeal swallowing than the thermal-tactile stimulation technique or complementary techniques.

Nevertheless, the effect of NMES treatment on dysphagia patients in this study did not show significant difference from

Table 1. Characteristics of the subjects

\begin{tabular}{lcc}
\hline Variables & $\begin{array}{c}\text { NMES } \\
(\mathrm{n}=24)\end{array}$ & $\begin{array}{c}\text { Masako maneuver } \\
(\mathrm{n}=23)\end{array}$ \\
\hline Gender (Male/Female) & $17 / 10$ & $16 / 12$ \\
Age (years)* & $67.9 \pm 5.6$ & $63.4 \pm 6.2$ \\
TDS (months) & $4.3 \pm 1.1$ & $4.5 \pm 1.2$ \\
FDS & $23.3 \pm 11.2$ & $24.1 \pm 11.8$ \\
\hline *p $<0.05$ & \multicolumn{3}{l}{ NMES: neuromuscular electrical stimulation; TSD: time since } \\
dysphagia; FDS: Functional Dysphagia Scale
\end{tabular}

Table 2. The result of analysis of pre-post values of FDS

\begin{tabular}{lcc}
\hline FDS & $\begin{array}{c}\text { NMES } \\
(\mathrm{n}=24)\end{array}$ & $\begin{array}{c}\text { Masako maneuver } \\
(\mathrm{n}=23)\end{array}$ \\
\hline Pre-test & $23.3 \pm 11.2$ & $24.1 \pm 11.8$ \\
Post-test & $18.3 \pm 6.8$ & $17.5 \pm 8.1$ \\
\hline
\end{tabular}


that of thermal-tactile stimulation. NMES treatment is frequently practiced in Korea on dysphagia caused by stroke ${ }^{12)}$. However, the use of NMES is restricted in pregnant patients with dysphagia or patients with a modulator for cardiac pacemaker ${ }^{13)}$. NMES also has less advantages than other swallowing rehabilitation techniques ${ }^{9)}$. Thus, care should be taken by rehabilitation experts in choosing their treatment methods.

The limitations of this study are as follows. First, all included subjects were patients with subacute. Second, the results of this study cannot be generalized for all dysphagia cases as they did not wear an L-tube. Hence, caution must be taken in interpreting the result of the study.

The Masako maneuver and NMES each showed significant effects on the improvement of swallowing function in patients with dysphagia caused by stroke, but no significant difference was observed between the two treatment methods. Randomized controlled trials are required in the future to verify the efficiency of NMES.

\section{REFERENCES}

1) Korea National Statistical Office: Cause of Death Statistics 2014, Daejeon: Korea National Statistical Office, 2015.

2) Higashijima M, Murata J, Ueda T, et al.: Clinical advantages of eating positions of the mid-neck on swallowing function. J Phys Ther Sci, 2012, 24: 837-840. [CrossRef]

3) Logemann JA: Evaluation and treatment of swallowing disorders. Austin: Pro-ed, 2002.

4) Won YS: Influence of manual facilitation technique on swallowing disorder and aspiration pneumonia caused by severe dysphagia with stroke. J Phys Ther Sci, 2012, 24: 909-913. [CrossRef]

5) Logemann JA: Treatment of oral and pharyngeal dysphagia. Phys Med Rehabil Clin N Am, 2008, 19: 803-816, ix. [Medline] [CrossRef]

6) Burkhead LM, Sapienza CM, Rosenbek JC: Strength-training exercise in dysphagia rehabilitation: principles, procedures, and directions for future research. Dysphagia, 2007, 22: 251-265. [Medline] [CrossRef]

7) Freed ML, Freed L, Chatburn RL, et al.: Electrical stimulation for swallowing disorders caused by stroke. Respir Care, 2001, 46: 466-474. [Medline]

8) Bülow M, Speyer R, Baijens L, et al.: Neuromuscular electrical stimulation (NMES) in stroke patients with oral and pharyngeal dysfunction. Dysphagia, 2008, 23: 302-309. [Medline] [CrossRef]

9) Kiger M, Brown CS, Watkins L: Dysphagia management: an analysis of patient outcomes using VitalStim therapy compared to traditional swallow therapy. Dysphagia, 2006, 21: 243-253. [Medline] [CrossRef]

10) Han TR, Back NJ, Park JW: The functional dysphagia scale using videofluoroscopic swallowing study in stroke patients. Ann Rehabil Med, 23: 1118-1126.

11) Clark H, Lazarus C, Arvedson J, et al.: Evidence-based systematic review: effects of neuromuscular electrical stimulation on swallowing and neural activation. Am J Speech Lang Pathol, 2009, 18: 361-375. [Medline] [CrossRef]

12) Wu HS, Jang KH, Cha TH et al.: A study of the status of dysphagia rehabilitation conducted by occupational therapists in Korea. J Kor Soc of Occup Ther, 17: 67-77.

13) Leelamanit V, Limsakul C, Geater A: Synchronized electrical stimulation in treating pharyngeal dysphagia. Laryngoscope, 2002, 112: 2204-2210. [Medline] [CrossRef] 